\title{
openheart Non-invasive assessment of functionally significant coronary stenoses through mathematical analysis of spectral ECG components
}

\author{
Tetsuya Amano, ${ }^{1}$ Norihiro Shinoda, ${ }^{2}$ Ayako Kunimura, ${ }^{2}$ Ken Harada, ${ }^{2}$ \\ Tadayuki Uetani, ${ }^{2}$ Hiroaki Takashima, ${ }^{1}$ Hirohiko Ando, ${ }^{1}$ Soichiro Kumagai, ${ }^{1}$ \\ Masahiko Gosho, ${ }^{3}$ Toyoaki Murohara ${ }^{4}$
}

To cite: Amano T, Shinoda N, Kunimura $A$, et al. Noninvasive assessment of functionally significant coronary stenoses through mathematical analysis of spectral ECG components. Open Heart 2014;1:e000144. doi:10.1136/openhrt-2014000144

Received 9 May 2014 Revised 26 August 2014 Accepted 4 November 2014

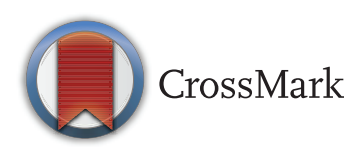

1Department of Cardiology, Aichi Medical University, Nagakute, Japan

${ }^{2}$ Department of Cardiology, Chubu Rosai Hospital, Nagoya, Japan

${ }^{3}$ Advanced Medical Research Center, Aichi Medical University, Nagakute, Japan ${ }^{4}$ Department of Cardiology, Nagoya University Graduate School of Medicine, Nagoya, Japan

Correspondence to Dr Tetsuya Amano: amanot@aichi-med-u.ac.jp

\section{ABSTRACT}

Objectives: The aim of this study was to evaluate the accuracy of the Multifunction CardioGram (MCG) in detecting the presence of functionally significant coronary ischaemia.

Methods and results: This prospective study evaluated the accuracy of the MCG, a new ECG analysis device used to diagnose ischaemic coronary artery disease (CAD). A consecutive 112 participants suspected to have CAD who were scheduled for elective coronary angiography (CAG) from October 2012 to December 2013 were examined. Their predictive values of relevant ischaemia were measured by MCG, standard ECG and Framingham Risk Score (FRS) and compared. Five levels of ischaemia based on CAG findings adjusted by fractional flow reserve (FFR) values and three levels of MCG score of high, borderline or low were used. The MCG (OR=2.67 (1.60 to 4.44$), p<0.001$ ) was the only test significantly associated with ischaemia level. The FFR values for individual MCG scores with low, borderline and high were 0.77 (0.70 to 0.86 ), 0.78 (0.71 to 0.82 ) and 0.69 (0.65 to 0.77 ), respectively, $p=0.042$. A high MCG score had a specificity of $90.4 \%$ (87.0\% to $93.9 \%)$ in model 1 adjusted by FFR $\leq 0.8$ threshold and of $87.0 \%$ $(83.2 \%$ to $90.8 \%$ ) in model 2 adjusted by FFR $\leq 0.75$ threshold, and a negative predictive value of $82.5 \%$ $(78.3 \%$ to $86.7 \%)$ in model 1 and of $83.8 \%(79.6 \%$ to $87.9 \%$ ) in model 2 for the prediction of severe ischaemia.

Conclusions: The MCG showed high specificity with a high negative predictive value, suggesting that the MCG could be used not only to identify functionally significant ischaemia but to reduce unnecessary CAGs. Trial registration number: UMIN ID: 000009992.

\section{INTRODUCTION}

Various types of cardiac stress tests, including ECG stress tests, nuclear scintigraphy and stress echocardiography, are standard noninvasive techniques for the evaluation of cardiac ischaemia. ${ }^{1-3}$ While these techniques

\section{KEY QUESTIONS}

What is already known about this subject?

- The Multifunction CardioGram (MCG) is a new computer-enhanced, multiphase, resting ECG analysis device that improves the quality of noninvasive tests. However, in previous trials that used MCG to detect the presence of relevant coronary artery disease, only a coronary angiography was the gold standard.

What does this study add?

- This prospective study was designed to evaluate the accuracy of MCG in diagnosing patients with coronary artery disease with functionally significant ischaemia defined by not only coronary angiography but fractional flow reserve reference standards.

How might this impact on clinical practice?

- The MCG might have the strength to identify functionally significant coronary ischaemia needing an optimal revascularisation.

are recognised as sensitive tests for the detection of coronary artery disease (CAD) in two or more large epicardial vessels, it is also acknowledged that they have a relatively poor specificity. There is a growing consensus that the poor specificity results in a significant number of unnecessary coronary angiographies (CAGs), thereby potentially subjecting many patients to the risks of invasive procedures and radiation exposure without commensurate clinical benefit. ${ }^{45}$

The Multifunction CardioGram (MCG) is a new computer-enhanced, multiphase, resting ECG analysis device that improves the quality of non-invasive tests. It has been used to determine the optimal decision-making algorithm for the evaluation of suspected obstructive CAD. ${ }^{6-9}$ The potential benefit of revascularisation depends on the presence of 
myocardial ischaemia, therefore careful identification of ischaemia-inducing stenosis allows for a greater benefit from revascularisation, especially in patients with stable angina pectoris (SAP) ${ }^{10-15}$ However, in previous trials that used MCG to detect the presence of relevant CAD, an immediate and subsequent CAG was the gold standard, without use of fractional flow reserve (FFR) ${ }^{6-9}$

The aim of this study, therefore, was to evaluate the accuracy of the MCG in detecting the presence of cardiac ischaemia by comparing its measurements with CAG and FFR in a relatively high-risk population who were scheduled for elective CAG, taking into account standard ECG and Framingham Risk Scores (FRS).

\section{METHODS}

\section{Patients and study design}

This prospective study was designed to evaluate the accuracy of MCG in diagnosing patients with CAD with severe ischaemia defined by CAG and FFR reference standards. Our target population was 112 consecutive participants with or without known CAD who were scheduled for elective CAG from October 2012 to December 2013. The exclusion criteria of this study were patients on haemodialysis $(n=2)$, those with elevated preprocedural cardiac biomarkers such as troponin- $T(n=5)$, prior coronary artery bypass graft $(\mathrm{CABG})$ surgery $(\mathrm{n}=2)$ and MCG of poor quality $(n=3)$. Thus, the final study population was 100 patients. This study was approved by the Institutional Review Board at Chubu Rosai hospital, all patients provided written informed consent, and the study was consistent with principles of the Declaration of Helsinki.

\section{Multifunctional cardiogram}

Each MCG test was taken and analysed prior to performing the CAG. An ECG was performed and $82 \mathrm{~s}$ of resting signal were collected from leads II and V5. The data were subsequently analysed and transformed by multiple mathematical functions (power spectra, cross correlation, coherence) resulting in a number of indices that were compared to a large database of patients with a broad range of clinically verified myocardial ischaemia. Test results were blinded to the angiographer. The MCG (Toray Medical Co Ltd) and associated computer with the MCG software V.2.1.1 (Premier Heart Japan Inc) were used. Three to five tests were taken at each session. Only those tests with marginal or better quality trace, which was checked automatically by the system, were sent for analysis to the PH LLC data centre through internet. The MCG device and database used have been previously described. ${ }^{16}$ In brief, the database against which the incoming MCG data are compared originated from data gathering trials conducted from 1978 to 2000 in more than 30 institutions in Europe, Asia and North America on 100 000 individuals of varying ages and degrees of coronary disease. The MCG reports also indicate the level of myocardial damage and severity integrated into a score; other information such as coronary damage, area of damage, and myocardial pathological and pathophysiological conditions are included in the report. An MCG score of four was used as the cut-off score in the majority of published clinical trials. ${ }^{9}{ }^{17}$ In the present study, we used a cut-off score of four but investigated the scoring method further. Patients were divided into three groups: high MCG score, minimum score among 3-5 tests at one session $\geq 4.0$; borderline MCG score, $4.0>$ all scores $\geq 3.0$; low MCG score, maximum score among 3-5 tests at one session $<3.0$.

\section{Standard ECG and FRS}

Specific ECG findings that were defined as positive were ST-segment depression, T-wave inversion and pathological $Q$ waves. ${ }^{18}{ }^{19}$ The FRS was also calculated for each case. ${ }^{20}{ }^{21}$ The patients were then categorised according to tertiles of risk score of high, intermediate and low.

\begin{tabular}{|c|c|}
\hline Variable & $\begin{array}{l}\text { Total } \\
\text { population } \\
(n=100)\end{array}$ \\
\hline \multicolumn{2}{|l|}{ Patient characteristics } \\
\hline Age, years & $69.5 \pm 9.5$ \\
\hline Male, n (\%) & $68(68)$ \\
\hline Diabetes mellitus, n (\%) & $40(40)$ \\
\hline Hypertension, n (\%) & 79 (79) \\
\hline Systolic blood pressure, $\mathrm{mm} \mathrm{Hg}$ & $132 \pm 14$ \\
\hline Dyslipidaemia, n (\%) & $77(77)$ \\
\hline HDL-cholesterol, mg/dL & $53 \pm 14$ \\
\hline Chronic kidney disease stage & $2.1 \pm 0.65$ \\
\hline Current Smoker, n (\%) & $24(24)$ \\
\hline Peripheral artery disease, $\mathrm{n}(\%)$ & $9(9)$ \\
\hline $\begin{array}{l}\text { History of stroke or transient ischaemic } \\
\text { attack, } \mathrm{n}(\%)\end{array}$ & $5(5)$ \\
\hline $\begin{array}{l}\text { History of percutaneous coronary } \\
\text { intervention, } n(\%)\end{array}$ & $23(23)$ \\
\hline History of myocardial infarction, n (\%) & $7(7)$ \\
\hline \multicolumn{2}{|l|}{ Angina, $\mathrm{n}(\%)$} \\
\hline Asymptomatic & $31(31)$ \\
\hline CCS class I & $32(32)$ \\
\hline CCS class II & $26(26)$ \\
\hline CCS class III & $11(11)$ \\
\hline Ejection fraction (\%) & $70.8 \pm 7.0$ \\
\hline Left ventricular hypertrophy, n (\%) & $28(28)$ \\
\hline Brain natriuretic peptide, $\mathrm{pg} / \mathrm{mL}$ & $26.1[15.9-48.4]$ \\
\hline $\mathrm{C}$ reactive protein, $\mathrm{mg} / \mathrm{L}$ & $1.2[0.5-3.4]$ \\
\hline ARB or $\mathrm{ACEI}, \mathrm{n}(\%)$ & $50(50)$ \\
\hline$\beta$-blocker, n (\%) & $20(20)$ \\
\hline Calcium channel blocker, n (\%) & 47 (47) \\
\hline Statins, n (\%) & 78 (78) \\
\hline
\end{tabular}


Table 2 Diagnosis tests and angiographic and FFR findings

\begin{tabular}{|c|c|}
\hline \multicolumn{2}{|l|}{ Diagnosis tests } \\
\hline \multicolumn{2}{|l|}{ Multifunction cardioGram score } \\
\hline High, n (\%) & $20(20)$ \\
\hline Borderline, n (\%) & $43(43)$ \\
\hline Low, n (\%) & 37 (37) \\
\hline \multicolumn{2}{|l|}{ ECG } \\
\hline Positive, n (\%) & $18(18)$ \\
\hline Negative, n (\%) & $82(82)$ \\
\hline \multicolumn{2}{|l|}{ Framingham Risk Score } \\
\hline High, n (\%) & $61(61)$ \\
\hline Intermediate, n (\%) & 34 (34) \\
\hline Low, n (\%) & $5(5)$ \\
\hline \multicolumn{2}{|l|}{ Revascularisation } \\
\hline PCl/CABG, n (\%) & $51(51) / 4(4)$ \\
\hline \multicolumn{2}{|l|}{ Lesion characteristics } \\
\hline Total number of lesions (>25\%) & 127 \\
\hline \multicolumn{2}{|l|}{ Angiographic findings } \\
\hline $\begin{array}{l}\text { Triple vessel disease or left main } \\
\text { disease }\end{array}$ & $17(13)$ \\
\hline Double vessel disease & $20(16)$ \\
\hline \multicolumn{2}{|l|}{ Stenosis, n (\%) } \\
\hline $26-50 \%$ of diameter & $24(19)$ \\
\hline $51-90 \%$ of diameter & $70(55)$ \\
\hline$>90 \%$ of diameter & $29(23)$ \\
\hline Total occlusion & $4(3)$ \\
\hline Collateral circulation & $14(11)$ \\
\hline \multicolumn{2}{|l|}{ FFR findings } \\
\hline Total number of vessels analysed & 121 \\
\hline Lesion with FFR $\leq 0.80$ & $57(47)$ \\
\hline Lesion with FFR $\leq 0.75$ & $43(36)$ \\
\hline
\end{tabular}

\section{CAG and FFR measurement}

Before performing a CAG, an intracoronary injection of $0.5 \mathrm{mg}$ isosorbide dinitrate was administered to prevent coronary spasm. Cineangiograms were analysed by an independent angiographer who was unaware of the MCG test results. FFR was calculated as previously described. ${ }^{13-15}$ In brief, equalisation was performed with the guide wire sensor positioned at the guiding catheter tip. The 0.014-inch pressure guide wire (St Jude Medical, Minneapolis, Minnesota, USA) was then advanced distally to the stenosis, and FFR was measured at maximal hyperaemia induced by intravenous ATP administered at $150 \mu \mathrm{g} / \mathrm{kg} / \mathrm{min}$ through a central or forearm vein. It was then calculated as the mean distal coronary pressure divided by the mean aortic pressure during maximal hyperaemia. Functional significance was defined as FFR values $\leq 0.80$ in model 1 and $\leq 0.75$ in model 2. The present study recommended that FFR should be measured in vessels that have intermediate stenosis (26-90\% of the vessel diameter). FFR measurements were deferred when vessels had obvious severe lesions $(>99 \%)$ with a delayed coronary flow or had no significant stenosis $(\leq 25 \%)$ as observed on CAG.

\section{Ischemic Severity Score}

Initially patients were categorised based on the percentage and location of stenosis estimated visually by CAG. ${ }^{22}{ }^{23}$ Five ischaemic grades were used; level 5 (severe), triple vessel disease or left main disease; level 4 (high), 99\% stenosis in a proximal lesion; level 3 (moderate), $75-90 \%$ stenosis in a proximal lesion, or $99 \%$ stenosis in a distal lesion; level 2 (mild), 75-90\% stenosis in a distal lesion; and level 1 (normal), stenosis of $50 \%$ or less. The location of stenosis was defined as the following: proximal for lesions in the right coronary artery (RCA) \#1-2, left anterior descending artery (LAD) \#67, left circumflex artery (LCX) \#11; distal for lesions in the RCA \#3-4, LAD \#8-10, LCX \#12-15. Next, the ischaemic grade was adjusted based on the FFR cut-off value of 0.80 as follows: levels 1 and 2 were adjusted to level 3 if the FFR was $\leq 0.80$ in model 1 or $\leq 0.75$ in model 2. Levels 5, 3 and 2 were adjusted to levels 3,2 and 1 , respectively, when the FFR was $>0.80$ in model 1 and $>0.75$ in model 2. Revascularisations such as percutaneous coronary intervention (PCI) and $\mathrm{CABG}$ were performed for patients with obvious severe stenosis $(>99 \%)$ or for lesions with the FFR $\leq 0.80$.

\section{Definition of clinical characteristics}

Diabetes mellitus was defined if the patient was taking any antihyperglycaemic medication or had previously been diagnosed with diabetes mellitus. Hypertensive patients were those with documented blood pressure of $>130 / 85 \mathrm{~mm} \mathrm{Hg}$ on two or more occasions, or who were already on antihypertensive therapy. A positive smoking status was defined if the patient currently smoked or had quit less than a year before entering the study. Chronic kidney disease stages were defined according to estimated glomerular filtration rate levels.

Table 3 Cumulative logit model analysis of three tests for prediction of ischaemia level (levels 1-5)

\begin{tabular}{|c|c|c|c|c|c|c|}
\hline \multirow[b]{2}{*}{ Test } & \multicolumn{3}{|c|}{ Model 1 (adjusted by FFR $\leq 0.8$ ) } & \multicolumn{3}{|c|}{ Model 2 (adjusted by FFR $\leq 0.75$ ) } \\
\hline & OR (95\% Cl) & p Value & AIC & OR (95\% Cl) & p Value & AIC \\
\hline MCG & 2.67 (1.60 to 4.44$)$ & $<0.001$ & 306.9 & 2.39 (1.44 to 3.94$)$ & $<0.001$ & 306.3 \\
\hline ECG & 2.15 (0.86 to 5.40$)$ & 0.10 & 317.8 & 1.87 (0.75 to 4.67$)$ & 0.18 & 315.6 \\
\hline FRS & 1.73 (0.94 to 3.17$)$ & 0.076 & 317.4 & 2.09 (1.13 to 3.88$)$ & 0.019 & 311.8 \\
\hline
\end{tabular}

AIC, Akaike information criterion; FFR, fractional flow reserve; FRS, Framingham Risk Sore; MCG, multifunction cardioGram. 
Figure 1 Proportion of

Multifunction CardioGram (MCG)

levels (low, borderline and high)

in each ischaemia level (level

1-5). The MCG level was

significantly associated with an

increasing level of ischaemia.

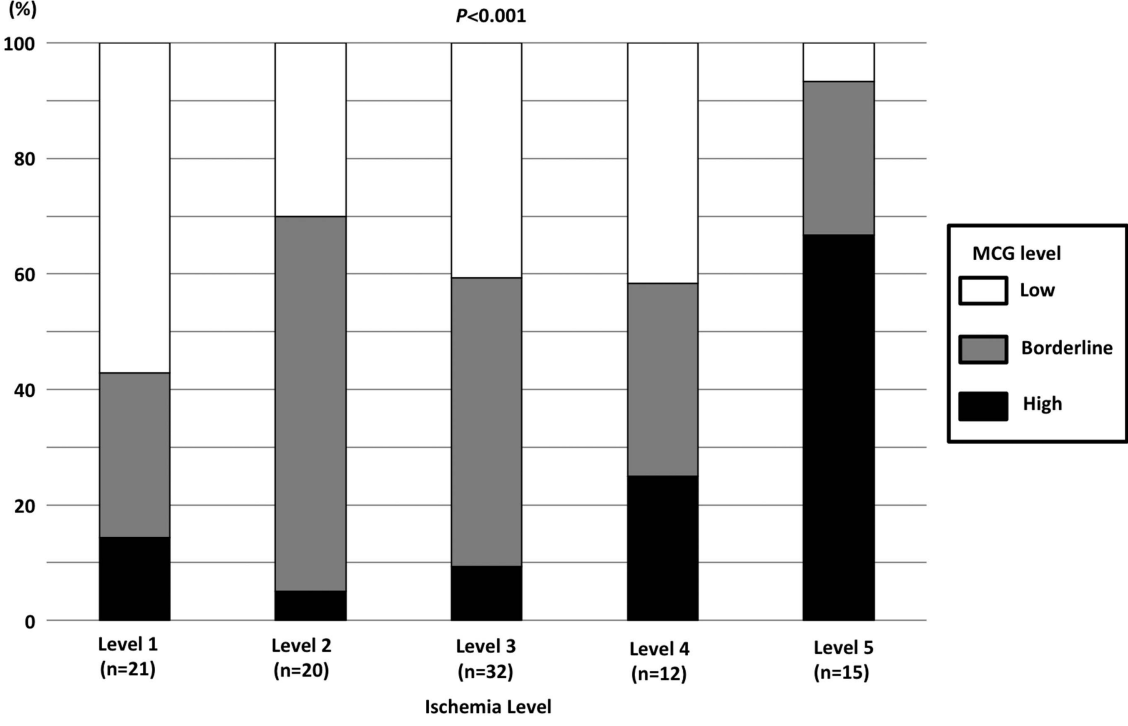

\section{Statistical analyses}

Categorical data were summarised as frequency (\%) and continuous data were expressed as mean and SD, or median and IQR as appropriate. For evaluating the relationship between the ischaemia level and three tests as predictors, the OR was calculated by applying a cumulative logit regression model on each of the three tests. We also conducted a logistic regression analysis to estimate the OR for the need for revascularisation. The Akaike Information Criterion (AIC) was used to compare the goodness of fit between the three models. Smaller AIC values indicate better fit. Dichotomised data were used to calculate the accuracy, sensitivity, specificity, and

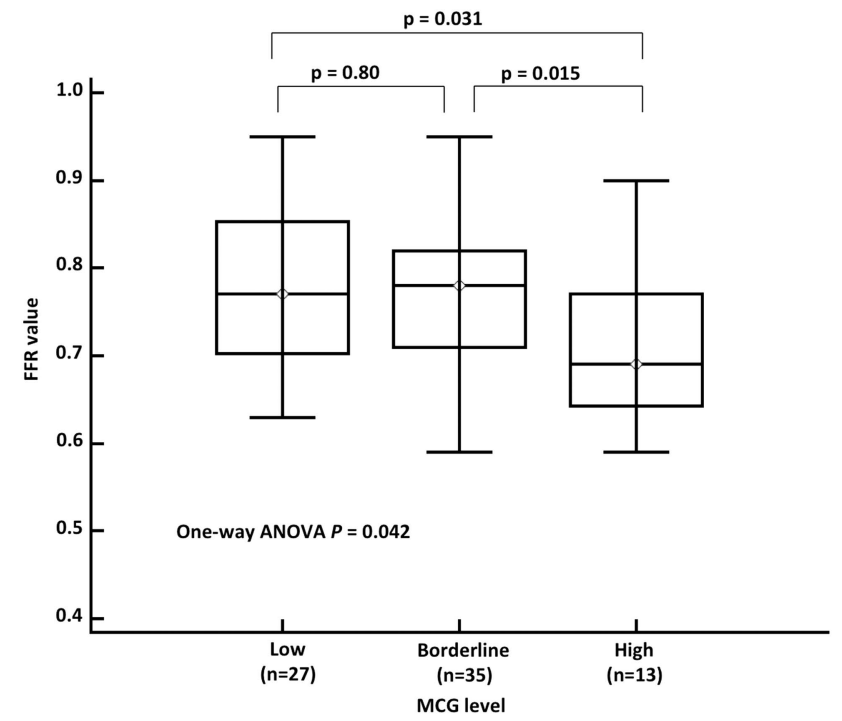

Figure 2 The fractional flow reserve (FFR) values according to increasing risks of the Multifunction CardioGram (MCG) scores. The FFR values were significantly associated with an increasing risk of the MCG score ( $p$ analysis of variance $(A N O V A)=0.042)$. The FFR values for individual MCG scores with low, borderline and high were 0.77 (0.70 to 0.86$), 0.78$ (0.71 to 0.82 ) and 0.69 (0.65 to 0.77 ), respectively. positive and negative predictive value (NPV) with a $95 \%$ CI. Agreement between the ischaemia level and three tests was evaluated by using Cohen's $\kappa$ coefficient. Sensitivity was calculated by dividing the number of patients in which a test was positive and in whom the ischaemia level was four or five (considered a true positive) by the total number of patients in which the ischaemia level was four or five. Specificity was calculated by dividing the number of patients in which a test is negative and the ischaemia level was one, two or three (considered a true negative) by the total number of patients in which the ischaemia level was one, two or three. Accuracy was calculated by dividing the number of patients with true positive or negatives by the total number of patients. We constructed two receiver operating curve (ROC) plots, one without taking the collateral cases into account and another with the collateral cases (all participants) to show the difference in MCG diagnostic accuracy. Three groupings of MCG scores (low, borderline and high) were evaluated using analysis of variance (ANOVA), and Fisher's least significant difference test for multiple comparisons to determine their associations with the FFR values was used with ANOVA. When the plural FFR values were available for one patient, the minimum FFR value was adopted. A value of $\mathrm{p}<0.05$ was considered statistically significant. All statistical analyses were performed with the SAS V.9.3 (SAS Institute, Cary, USA) software.

\section{RESULTS}

\section{Baseline characteristics}

Table 1 outlines the baseline clinical characteristics of all 100 patients. Forty per cent of patients had diabetes. Nearly $60 \%$ of patients had class I and II angina as measured by the Canadian Cardiovascular Society scale. Thirty-one per cent of patients were asymptomatic. Nearly $80 \%$ of patients were taking statin therapy. Table 2 shows diagnosis test, angiographic and FFR findings. Twenty patients (20\%) had a high MCG score. 
Table 4 Predictive value of severe ischaemia (levels 4 and 5) by MCG, ECG and FRS

\begin{tabular}{|c|c|c|c|c|c|c|c|}
\hline Test & к Coefficient $(95 \% \mathrm{Cl})$ & p Value & Accuracy & Sensitivity & Specificity & PPV & NPV \\
\hline \multicolumn{8}{|c|}{ (A) Model 1 (adjusted by FFR $\leq 0.8$ ) } \\
\hline \multicolumn{8}{|l|}{ All patients } \\
\hline MCG (H vs B/L) & $0.42(0.22$ to 0.62$)$ & - & 79.0 (74.9 to 83.1$)$ & 48.1 (38.5 to 57.8$)$ & 90.4 (87.0 to 93.9$)$ & 65.0 (54.3 to 75.7$)$ & 82.5 (78.3 to 86.7$)$ \\
\hline ECG (P vs $N)$ & $0.12(-0.08$ to 0.33$)$ & 0.020 & 69.0 (64.4 to 73.6$)$ & 25.9 (17.5 to 34.4$)$ & 84.9 (80.7 to 89.1$)$ & 38.9 (27.4 to 50.4$)$ & 75.6 (70.9 to 80.4$)$ \\
\hline FRS ( $\mathrm{H}$ vs I/L) & $0.16(0.02$ to 0.31$)$ & 0.024 & $54.0(49.0$ to 59.0$)$ & $77.8(69.8$ to 85.8$)$ & $45.2(39.4$ to 51.0$)$ & 34.4 (28.3 to 40.5$)$ & 84.6 (78.8 to 90.4$)$ \\
\hline \multicolumn{8}{|l|}{ Male } \\
\hline MCG (H vs B/L) & $0.40(0.16$ to 0.64$)$ & - & 76.5 (71.3 to 81.6$)$ & 47.6 (36.7 to 58.5$)$ & 89.4 (84.9 to 93.9$)$ & 66.7 (54.3 to 78.8$)$ & 79.2 (73.7 to 84.8$)$ \\
\hline ECG (P vs N) & $0.07(-0.1$ to 0.30$)$ & 0.027 & 66.2 (60.4 to 71.9$)$ & 19.0 (10.5 to 27.6$)$ & 87.2 (82.4 to 92.1$)$ & 40.0 (24.5 to 55.5$)$ & 70.7 (64.7 to 76.7$)$ \\
\hline FRS (H vs I/L) & $0.05(-0.08$ to 0.18$)$ & 0.001 & 41.2 (35.2 to 47.1$)$ & 85.7 (78.1 to 93.4$)$ & 21.3 (15.3 to 27.2$)$ & 32.7 (26.4 to 39.1$)$ & 76.9 (65.2 to 88.6$)$ \\
\hline \multicolumn{8}{|l|}{ Female } \\
\hline MCG (H vs B/L) & 0.45 (0.05 to 0.86$)$ & - & 84.4 (78.0 to 90.8$)$ & 50.0 (29.6 to 70.4$)$ & 92.3 (87.1 to 97.5$)$ & 60.0 (38.1 to 81.9$)$ & 88.9 (82.8 to 94.9$)$ \\
\hline ECG (P vs N) & $0.27(-0.11$ to 0.65$)$ & 0.77 & 75.0 (67.3 to 82.7$)$ & 50.0 (29.6 to 70.4$)$ & 80.8 (73.0 to 88.5$)$ & 37.5 (20.4 to 54.6$)$ & 87.5 (80.7 to 94.3$)$ \\
\hline FRS (H vs I/L) & $0.38(-0.02$ to 0.79$)$ & 0.94 & 81.3 (74.4 to 88.1$)$ & $50.0(29.6$ to 70.4$)$ & 88.5 (82.2 to 94.7$)$ & $50.0(29.6$ to 70.4$)$ & 88.5 (82.2 to 94.7$)$ \\
\hline \multicolumn{8}{|c|}{ (B) Model 2 (adjusted by FFR $\leq 0.75$ ) } \\
\hline \multicolumn{8}{|l|}{ All patients } \\
\hline MCG (H vs B/L) & $0.32(0.10$ to 0.54$)$ & - & 77.0 (72.8 to 81.2$)$ & 43.5 (33.1 to 53.8$)$ & 87.0 (83.2 to 90.8$)$ & 50.0 (38.8 to 61.2$)$ & 83.8 (79.6 to 87.9$)$ \\
\hline$E C G(P$ vs $N)$ & $0.05(-0.15$ to 0.26$)$ & 0.066 & 69.0 (64.4 to 73.6$)$ & 21.7 (13.1 to 30.3$)$ & 83.1 (78.8 to 87.4$)$ & 27.8 (17.2 to 38.3 ) & 78.0 (73.5 to 82.6$)$ \\
\hline FRS (H vs I/L) & $0.14(0.01$ to 0.28$)$ & 0.24 & 52.0 (47.0 to 57.0 ) & 78.3 (69.7 to 86.9$)$ & 44.2 (38.5 to 49.8$)$ & 29.5 (23.7 to 35.5 ) & 87.2 (81.8 to 92.5$)$ \\
\hline \multicolumn{8}{|l|}{ Male } \\
\hline MCG (H vs B/L) & 0.38 (0.13 to 0.62$)$ & - & 76.5 (71.3 to 81.6$)$ & 47.4 (35.9 to 58.8 ) & 87.8 (83.1 to 92.4$)$ & 60.0 (47.4 to 72.6$)$ & 81.1 (75.8 to 86.5$)$ \\
\hline ECG (P vs N) & $0.02(-0.21$ to 0.24$)$ & 0.014 & 66.2 (60.4 to 71.9$)$ & 15.8 (7.4 to 24.2$)$ & 85.7 (80.7 to 90.7 ) & 30.0 (15.5 to 44.5$)$ & 72.4 (66.5 to 78.3$)$ \\
\hline FRS ( $\mathrm{H}$ vs I/L) & $0.03(-0.10$ to 0.16$)$ & 0.002 & 38.2 (32.3 to 44.1 ) & 84.2 (75.8 to 92.6$)$ & 20.4 (14.7 to 26.2$)$ & 29.1 (23.0 to 35.2 ) & 76.9 (65.2 to 88.6$)$ \\
\hline \multicolumn{8}{|l|}{ Female } \\
\hline MCG (H vs B/L) & $0.10(-0.30$ to 0.50$)$ & - & 78.1 (70.8 to 85.4$)$ & 25.0 (3.3 to 46.7$)$ & 85.7 (79.1 to 92.3 ) & 20.0 (2.1 to 37.9$)$ & 88.9 (82.8 to 94.9$)$ \\
\hline ECG (P vs $N)$ & $0.20(-0.17$ to 0.57$)$ & 0.90 & 75.0 (67.3 to 82.7$)$ & 50.0 (25.0 to 75.0$)$ & 78.6 (70.8 to 86.3 ) & 25.0 (9.7 to 40.3$)$ & 91.7 (86.0 to 97.3 ) \\
\hline FRS (H vs I/L) & $0.29(-0.13$ to 0.72$)$ & 0.75 & 81.3 (74.4 to 88.1 ) & 50.0 (29.6 to 70.4$)$ & 88.5 (82.2 to 94.7$)$ & 50.0 (29.6 to 70.4$)$ & 92.3 (87.1 to 97.5$)$ \\
\hline
\end{tabular}

FRS, Framingham Risk Sore; H vs B/L, high versus borderline/low; $\mathrm{H}$ vs I/L, high versus intermediate/low; MCG, multifunction cardioGram; NPV, negative predictive value; PPV, positive

predictive value; $\mathrm{P}$ vs $\mathrm{N}$, positive versus negative. 

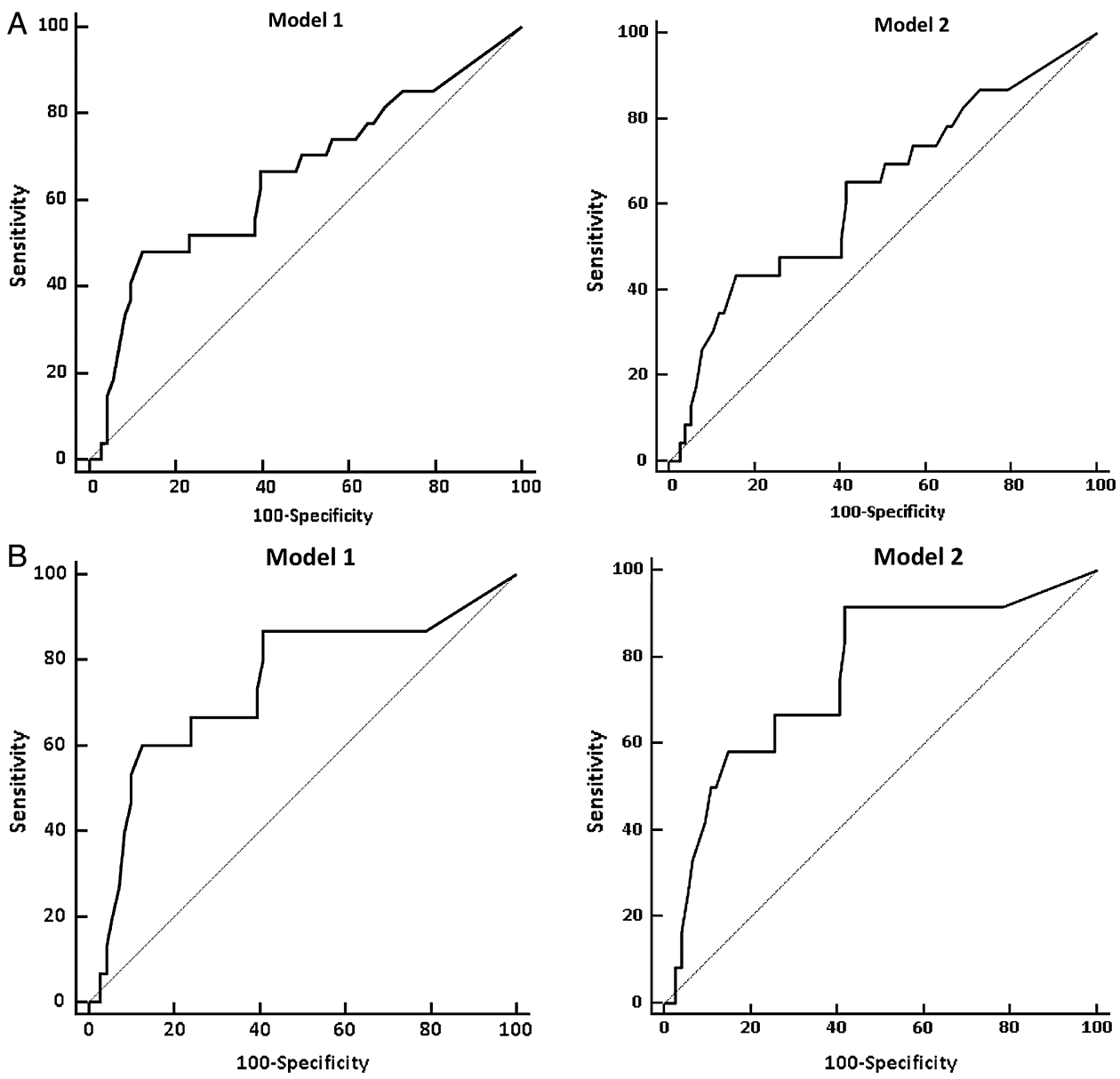

Figure 3 (A) ROC analyses of the MCG score for the prediction of severe ischaemia (levels 4 and 5) in all participants. The area under the receiver operating curve (ROC) for the Multifunction CardioGram (MCG) score in prediction of severe ischaemia was 0.66 ( 0.55 to 0.75$)$ in model 1 and 0.63 ( 0.53 to 0.73 ) in model 2 . The optimal cut-off value identified by ROC analysis was 4.8 in model 1 and model 2 with a sensitivity of $48.1 \%$ and a specificity of $87.7 \%$ in model 1 and a sensitivity of $43.5 \%$ and a specificity of $84.4 \%$ in model 2. (B) ROC analyses of the MCG score for the prediction of severe ischaemia (levels 4 and 5 ) in patients without collateral circulation. When these patients are excluded from analysis, the area under the ROC for the MCG score increased to 0.74 (0.64 to 0.83 ) in model 1 and 0.76 (0.66 to 0.85 ) in model 2 . The optimal cut-off value, sensitivity and specificity for the prediction of severe ischaemia were $4.8,60.0 \%$ and $87.3 \%$ in model 1 , and $3.0,91.7 \%$ and $58.1 \%$ in model 2 , respectively.

There were a total of 127 lesions $(>25 \%$ of diameter) in 89 patients $(89 \%)$, and $11(11 \%)$ had no significant stenosis. The collateral circulations were observed in 14 lesions $(11 \%)$ with 14 patients $(14 \%)$. The FFR was measured in 75 patients $(75 \%)$, in a total of 121 vessels. There were 57 lesions $(47 \%)$ with an FFR value $<0.80$. Revascularisations (PCI or CABG) were performed in 55 $(55 \%)$ of all patients.

\section{Association between MCG, ECG and FRS and ischaemia levels}

Table 3 shows the cumulative logit model analysis of the three tests for prediction of ischaemia levels. The MCG $(\mathrm{OR}=2.67$ (1.60 to 4.44), $\mathrm{p}<0.001$ in model 1 and $\mathrm{OR}=2.39$ (1.44 to 3.94$), \mathrm{p}<0.001$ in model 2 ) was the only test significantly associated with ischaemia level. As shown in figure 1, the MCG level (low, borderline and high) was significantly associated with an increasing level of ischaemia (level 1-5). Figure 2 shows the FFR values for individual MCG scores with low, borderline and high. The FFR values were significantly associated with an increasing risk of the MCG score ( $p$ ANOVA=0.042). Table 4 shows the predictive values of severe ischaemia (level 4 and 5) by MCG (high vs borderline/low), ECG (positive vs negative) and FRS (high vs intermediate/ low). A high MCG score $(\geq 4.0)$ had a specificity of $90.4 \%(87.0 \%$ to $93.9 \%)$ in model 1 adjusted by FFR $\leq 0.8$ (table $4 \mathrm{~A})$, and of $87.0 \%(83.2 \%$ to $90.8 \%)$ in model 2 adjusted by FFR $\leq 0.75$ (table $4 \mathrm{~B}$ ), and a NPV of $82.5 \%(78.3 \%$ to $86.7 \%)$ in model 1 and of $83.8 \%$ (79.6\% to $87.9 \%$ ) in model 2 for the prediction of severe ischaemia. In examining the $\kappa$ coefficient in all patients, the MCG (0.42 (0.22 to 0.62)) was significantly superior to the ECG $(0.12$ ( -0.08 to 0.33$), \mathrm{p}=0.020)$ and FRS $(0.16(0.02$ to 0.31$), \mathrm{p}=0.024)$ in model 1 . The MCG showed a relatively high predictive accuracy of 
A

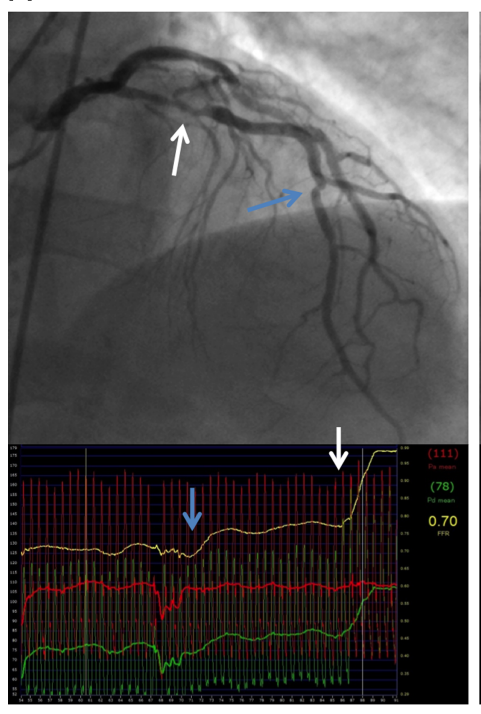

B

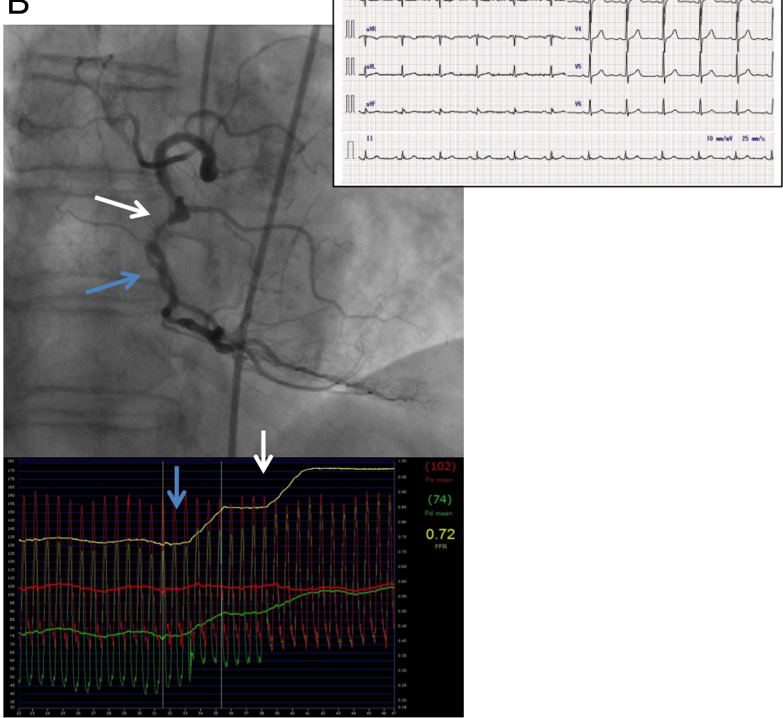

Figure 4 Representative images of coronary angiography and fractional flow reserve (FFR) from a patient who showed no resting ECG abnormality. The average Multifunction CardioGram value of this patient was 6.0, and two vessel disease in the proximal left anterior descending artery $(F F R=0.70)$ in panel $A$, and the mid right coronary artery $(F F R=0.72)$ in panel $B$ were found. Arrows show the jump up points of FFR.

approximately $80 \%$, irrespective of gender. The area under the ROC (AUC) for the MCG score in prediction of severe ischaemia was 0.66 (0.55 to 0.75$)$ in model 1 and 0.63 (0.53 to 0.73 ) in model 2. The optimal cut-off value identified by ROC analysis was 4.8 in model 1 and model 2, with a sensitivity of $48.1 \%$ and a specificity of $87.7 \%$ in model 1 , and a sensitivity of $43.5 \%$ and a specificity of $84.4 \%$ in model 2 (figure $3 \mathrm{~A}$ ). In our study, there were 14 patients having a well-established collateral circulation. When these patients were excluded from analysis, the AUC for the MCG score increased to 0.74 (0.64 to 0.83 ) in model 1 and 0.76 (0.66 to 0.85$)$ in model 2. The optimal cut-off value, sensitivity and specificity for the prediction of severe ischaemia were 4.8 , $60.0 \%$ and $87.3 \%$ in model 1 and $3.0,91.7 \%$ and $58.1 \%$ in model 2, respectively (figure 3B). Figure 4 shows the representative images of CAG and FFR from a patient who showed no resting ECG abnormality. The average MCG value of this patient was 6.0, and two vessel disease in the proximal $\mathrm{LAD}(\mathrm{FFR}=0.70)$ and the mid $\mathrm{RCA}$ (FFR=0.72) were found.

\section{DISCUSSION}

In the present study, the MCG, a new computerenhanced, multiphase, resting ECG analysis device, was significantly associated with the severity of coronary ischaemia as determined by CAG and FFR, in a relatively high-risk population with or without known CAD. All patients were scheduled for an elective CAG, and the MCG showed a relatively high predictive accuracy of approximately $80 \%$, irrespective of gender.
Recently, Patel et $a l^{24}$ published an analysis of the American College of Cardiology National Cardiovascular Data Registry, which included 397954 patients without known $\mathrm{CAD}$ who were undergoing elective CAG. CAD was absent in $39.2 \%$ of these patients. The authors created four separate models for the prediction of positive results in CAG: (1) FRS alone; (2) FRS plus clinical factors; (3) FRS, clinical factors and presence of symptoms; and (4) results of non-invasive testing. They concluded that although a positive non-invasive test was associated with the presence of obstructive $\mathrm{CAD}$, the addition of information obtained from non-invasive tests had a limited effect on the model's predictive ability over and above the effect achieved from the addition of clinical risk factors and symptoms. This large and important study clearly identifies the limitations of non-invasive testing in selecting patients most likely to benefit from CAG. The diagnostic performance compares favourably to other noninvasive diagnostic tests. A review of stress scintigraphy studies reported a wide range of sensitivities from $44 \%$ to $89 \%$ and specificities of $89-94 \%$ for two vessel disease. ${ }^{25}$ Numerous studies of exercise ECG as a diagnostic tool for CAD have been conducted, and reported sensitivities range from $31 \%$ to $\geq 90 \%$, while specificities range from $46 \%$ to nearly $100 \%$.

The data presented in this study on sensitivity, specificity and NPV of $48.1 \%, 90.4 \%$ and $82.5 \%$, respectively, for the detection of severe ischaemia are considerably equal or superior to those of the most widely used stress ECG and stress perfusion imaging. These relatively high 
specificities confirm the strength of the MCG device in reducing unnecessary CAGs. Additionally, the use of MCG in clinical practice could be reliably extended to patients who have difficulty in exercising and who have economic limitations.

In our study, ROC analyses excluding patients with a well-established collateral circulation improved the sensitivity of the prediction of severe ischaemia from $48.1 \%$ to $60.0 \%$. This finding suggests the limitation of the MCG device in situations of critical stenosis in an epicardial vessel with a well-established circulation. This may result in a reduction in myocardial ischaemia, leading to a false-negative result. More importantly, using these unique categorisations of patients will lead to a better understanding of their pathology. Further studies are needed in this regard.

In the most recent trial, the MCG has been shown to safely and accurately identify patients with relevant coronary stenosis determined by CAG alone. ${ }^{6-9}$ In cases of PCI in patients with SAP, careful identification of ischaemia-inducing stenosis is essential for obtaining the greater benefit from revascularisation. ${ }^{10-13}$ In other words, the information from functionally significant ischaemia in addition to CAG findings provides a potential benefit in determining the indication of PCI, especially in patients with SAP.

\section{LIMITATIONS}

The limitation of the present study is the recruitment of patients. The patients in this study represented a relatively high-risk population with or without known $\mathrm{CAD}$ who were scheduled for elective CAG; this may limit the generalisation of our results. However, FFR used during CAG was performed for an intermediate lesion to determine the functional ischaemia, thus, recruitment of patients who did not plan a CAG was an ethical dilemma. In addition, this study assessed the accuracy of the MCG by use of the FFR and used the ischaemic severity score as a reference for functional ischaemia. However, prognostic impact of the reference for ischaemia used remained elusive. Further study is needed in this regard.

\section{CONCLUSIONS}

In conclusion, the MCG showed a high specificity and high NPV regardless of gender in relatively high-risk patients, suggesting that the MCG could identify relevant severe ischaemia. In addition, the potential use of MCG in the evaluation of ischemic CAD appeared to be more feasible than standard ECG and FRS.

\section{Competing interests None.}

Patient consent Obtained.

Ethics approval Obtained

Provenance and peer review Not commissioned; externally peer reviewed.

Data sharing statement No additional data are available.
Open Access This is an Open Access article distributed in accordance with the Creative Commons Attribution Non Commercial (CC BY-NC 4.0) license, which permits others to distribute, remix, adapt, build upon this work noncommercially, and license their derivative works on different terms, provided the original work is properly cited and the use is non-commercial. See: http:// creativecommons.org/licenses/by-nc/4.0/

\section{REFERENCES}

1. Gibbons RJ, Balady GJ, Bricker JT, et al. Acc/aha 2002 guideline update for exercise testing: summary article: a report of the American college of cardiology/American heart association task force on practice guidelines (committee to update the 1997 exercise testing guidelines). Circulation 2002;106:1883-92.

2. Hecht HS, Shaw RE, Chin HL, et al. Silent ischemia after coronary angioplasty: evaluation of restenosis and extent of ischemia in asymptomatic patients by tomographic thallium-201 exercise imaging and comparison with symptomatic patients. J Am Coll Cardiol 1991;17:670-7.

3. Pirelli S, Danzi GB, Alberti A, et al. Comparison of usefulness of high-dose dipyridamole echocardiography and exercise electrocardiography for detection of asymptomatic restenosis after coronary angioplasty. Am J Cardiol 1991;67:1335-8.

4. Scanlon PJ, Faxon DP, Audet AM, et al. Acc/aha guidelines for coronary angiography: executive summary and recommendations. A report of the American college of cardiology/American heart association task force on practice guidelines (committee on coronary angiography) developed in collaboration with the society for cardiac angiography and interventions. Circulation 1999;99:2345-57.

5. Budoff MJ, Achenbach S, Duerinckx A. Clinical utility of computed tomography and magnetic resonance techniques for noninvasive coronary angiography. J Am Coll Cardiol 2003;42:1867-78.

6. Weiss MB, Narasimhadevara SM, Feng GQ, et al. Computer-enhanced frequency-domain and 12-lead electrocardiography accurately detect abnormalities consistent with obstructive and nonobstructive coronary artery disease. Heart Dis 2002;4:2-12.

7. Grube E, Bootsveld A, Buellesfeld L, et al. Computerized two-lead resting ecg analysis for the detection of coronary artery stenosis after coronary revascularization. Int J Med Sci 2008;5:50-61.

8. Hosokawa J, Shen JT, Imhoff M. Computerized 2-lead resting ecg analysis for the detection of relevant coronary artery stenosis in comparison with angiographic findings. Congest Heart Fail 2008;14:251-60.

9. Strobeck JE, Shen JT, Singh B, et al. Comparison of a two-lead, computerized, resting ecg signal analysis device, the multifunction-cardiogram or mcg (a.K.A. 3dmp), to quantitative coronary angiography for the detection of relevant coronary artery stenosis $(>70 \%)$ - a meta-analysis of all published trials performed and analyzed in the us. Int $J$ Med Sci 2009;6:143-55.

10. Hachamovitch R, Hayes SW, Friedman JD, et al. Comparison of the short-term survival benefit associated with revascularization compared with medical therapy in patients with no prior coronary artery disease undergoing stress myocardial perfusion single photon emission computed tomography. Circulation 2003;107:2900-7.

11. Pijls $\mathrm{NH}$, van Schaardenburgh $\mathrm{P}$, Manoharan $\mathrm{G}$, et al. Percutaneous coronary intervention of functionally nonsignificant stenosis: 5-year follow-up of the defer study. J Am Coll Cardiol 2007;49:2105-11.

12. Shaw LJ, Berman DS, Maron DJ, et al. Optimal medical therapy with or without percutaneous coronary intervention to reduce ischemic burden: results from the clinical outcomes utilizing revascularization and aggressive drug evaluation (courage) trial nuclear substudy. Circulation 2008;117:1283-91.

13. De Bruyne B, Pijls NH, Kalesan B, et al. Fractional flow reserve-guided pci versus medical therapy in stable coronary disease. N Engl J Med 2012;367:991-1001.

14. Pijls NH, van Son JA, Kirkeeide RL, et al. Experimental basis of determining maximum coronary, myocardial, and collateral blood flow by pressure measurements for assessing functional stenosis severity before and after percutaneous transluminal coronary angioplasty. Circulation 1993;87:1354-67.

15. Pijls NH, De Bruyne B, Peels K, et al. Measurement of fractional flow reserve to assess the functional severity of coronary-artery stenoses. N Engl J Med 1996;334:1703-8.

16. Strobeck JE, Mangieri A, Rainford N. A paired-comparision of the multifunction cardiogram $(\mathrm{mcg})$ and sestamibi spect myocardial perfusion imaging (mpi) to quantitative coronary angiography for the detection of relevant coronary artery obstruction $(>/=70 \%)-a$ single-center study of 116 consecutive patients referred for coronary angiography. Int J Med Sci 2011;8:717-24. 
17. Grube E, Bootsveld A, Yuecel S, et al. Computerized two-lead resting ecg analysis for the detection of coronary artery stenosis. Int J Med Sci 2007;4:249-63.

18. Ashley EA, Raxwal V, Froelicher V. An evidence-based review of the resting electrocardiogram as a screening technique for heart disease. Prog Cardiovasc Dis 2001;44:55-67.

19. Larsen CT, Dahlin J, Blackburn $\mathrm{H}$, et al. Prevalence and prognosis of electrocardiographic left ventricular hypertrophy, st segment depression and negative t-wave; the copenhagen city heart study. Eur Heart J 2002;23:315-24.

20. D'Agostino RB Sr, Vasan RS, Pencina MJ, et al. General cardiovascular risk profile for use in primary care: the framingham heart study. Circulation 2008;117:743-53.
21. Expert Panel on Detection, Evaluation, and Treatment of High Blood Cholesterol in Adults. Executive summary of the third report of the national cholesterol education program (ncep) expert panel on detection, evaluation, and treatment of high blood cholesterol in adults (adult treatment panel iii). JAMA 2001;285:2486-97.

22. Gensini GG. Coronary arteriography. New York: Futura Press, 1975.

23. Gensini GG. A more meaningful scoring system for determining the severity of coronary heart disease. Am J Cardiol 1983;51:606.

24. Patel MR, Peterson ED, Dai D, et al. Low diagnostic yield of elective coronary angiography. N Engl J Med 2010;362:886-95.

25. Elhendy A, Bax JJ, Poldermans D. Dobutamine stress myocardial perfusion imaging in coronary artery disease. J Nucl Med 2002;43:1634-46. 